\title{
A new approach for blocking KRAS
}

A rational drug design approach has yielded potent small-molecule compounds that directly disrupt the signalling of oncogenic KRAS through a new target: phosphodiesterase $\delta$-subunit (PDE $\delta$ ). KRAS is frequently mutated in many cancers and has been the focus of substantial drug discovery efforts, but so far no KRAS inhibitors have been successful in clinical trials.

$\mathrm{PDE} \delta$ is a protein that binds to and actively shuttles newly processed KRAS to the correct location in plasma membranes, where it can then activate downstream effectors. As the distribution of KRAS to particular locations of the cell membrane is crucial for its oncogenic activity, Zimmermann and colleagues proposed that disruption of the KRAS-PDE $\delta$ interaction would then abrogate oncogenic signalling by KRAS.

A high-throughput screen was first used to identify small molecules that bind to the farnesylbinding pocket of PDE $\delta$. This binding pocket is where farnesylated KRAS - a post-translationally modified form of KRAS that helps KRAS to associate with the plasma membrane - interacts with PDE $\delta$. Several benzimidazole hits were identified, which were then further characterized and derivatives generated through structure-guided design. The derivative that had the most favourable pharmacological properties (nanomolar potency as well as sufficient solubility and membrane permeability) was subsequently used for further studies.

Fluorescence microscopy studies confirmed that this derivative, named deltarasin, directly disrupted the KRAS-PDE $\delta$ interaction. In two human pancreatic ductal adenocarcinoma cell lines (PANC-1 and Panc-Tu-I), addition of $5 \mu \mathrm{M}$ of deltarasin led to the redistribution of KRAS from a localized area in the plasma membrane to a more random arrangement to the endomembranes. In cell lines that were dependent on oncogenic KRAS for survival (Panc-Tu-I cells and Capan-1 cells) this redistribution of KRAS led to a strong reduction in cell proliferation and increased cell death in a dosedependent manner. Conversely, in cells that were not dependent on oncogenic KRAS (PANC-1 cells), the addition of deltarasin had a minimal effect on cell survival and growth.
The disruption in KRAS localization also affected its signalling capacity, as reduced levels of the downstream kinases extracellular signal-regulated kinase 1 (ERK1) and ERK2 were seen in Panc-Tu-I cells and in Capan-1 cells treated with deltarasin. Finally, in nude mice bearing subcutaneous xenografts of human Panc-Tu-I tumours, intraperitoneal administration of deltarasin once or twice a day led to dose-dependent reductions in tumour growth.

Overall, these small molecules represent a platform on which further direct inhibitors of oncogenic KRAS signalling could be developed.

Man Tsuey Tse

ORIGINAL RESEARCH PAPER Zimmermann, G et al. Small molecule inhibition of the KRAS-PDE $\delta$ interaction impairs oncogenic KRAS signalling. Nature 497, 638-642 (2013)

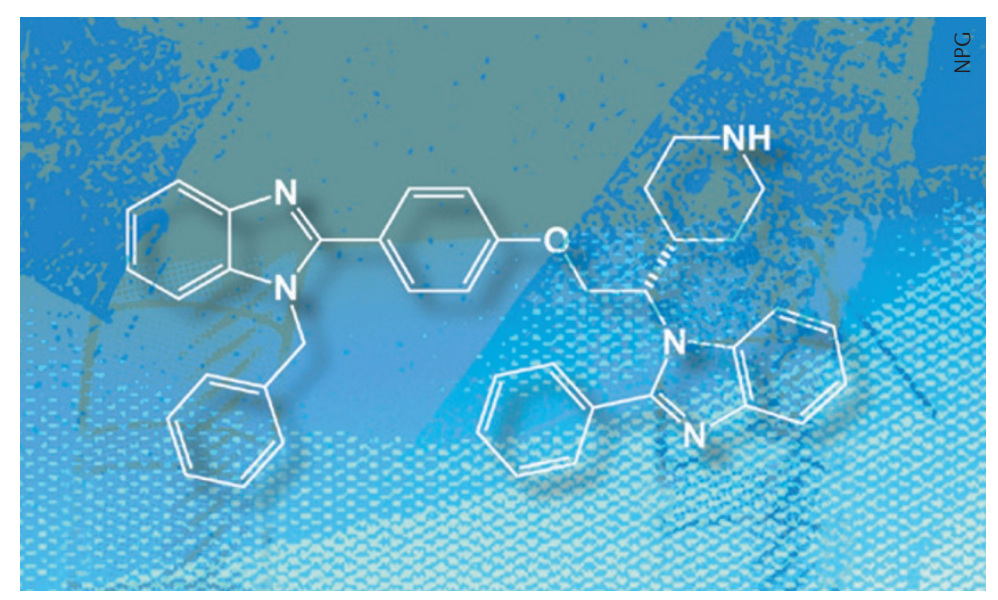

Structure of deltarasin $\left(K_{d} 38 \pm 16 \mathrm{nM}\right)$, a novel PDE $\delta$ inhibitor that disrupts oncogenic KRAS signalling. 